\title{
The diagnostic value of diffusion-weighted imaging in differentiating benign from malignant hepatic lesions
}

\author{
Reza Javadrashid ${ }^{1}$, Abolhassan Shakeri Bavil Olyaei ${ }^{1}$, Mohammad Kazem Tarzamni $^{1}$, Roghayeh Razzaghi ${ }^{1}$, \\ Javad Jalili', Shahryar Hashemzadeh², Mohammad Mirza-Aghazadeh-Attari ${ }^{3}$, Ali Kiani Nazarlou ${ }^{4}$ and \\ Armin Zarrintan ${ }^{4^{*}}$
}

\begin{abstract}
Background: Diffusion-weighted imaging (DWI) is a novel imaging technique with growing application in oncoimaging. This modality evaluates the diffusion of water molecules in various tissues, which is restricted in hyper cellular regions such as malignant tissue. Apparent diffusion co-efficient (ADC) is a method which can quantify the degree of restriction in tissues and can have diagnostic roles in characterization of hepatic lesions. In this study, 93 patients with proven hepatic lesions were included. These patients had undergone initial evaluation via ultrasonography and dynamic $C T$ scan, and had a definite diagnosis confirmed by biopsy. These patients underwent DW imaging and ADC values of their lesions were calculated. Patients were divided into two groups, benign and malignant groups, based on their biopsy results; and ADC values of hepatic lesions were compared in the two groups.

Results: The two groups were gender matched. There was a significant difference in the age distribution between the two groups. Mean ADC values for benign and malignant hepatic lesions were $1.58 \pm 0.35\left(10^{-3} \mathrm{~mm}^{2} / \mathrm{s}\right)$ and 0.87 $\pm 0.16\left(10^{-3} \mathrm{~mm}^{2} / \mathrm{s}\right)$, respectively. There was a statistically significant differences between benign and malignant hepatic lesions $\left(p\right.$ value $<10^{-3}$ ). DW imaging had a sensitivity of $97.6 \%$ and specificity of $98.7 \%$ in detecting malignant hepatic lesions from benign ones ( $p=0.0001$, AUC $=0.99$ ).

Conclusion: DW MRI imaging can differentiate malignant and benign liver lesions with high sensitivity and specificity using ADC values generated; furthermore, each subgroup of hepatic lesions could be determined based on ADC values.
\end{abstract}

Keywords: (DWI) Diffusion weighted, Hepatic mass, MRI

\section{Background}

Diffusion-weighted (DW) magnetic resonance imaging (MRI) was initially introduced in the middle of the 1990s and has since gained clinical significance in human pathologies. This imaging technique relies on variances in diffusion of water molecules in intracellular and intercellular spaces [1]. Initial efforts were made to utilize DW-MRI in stroke patients, as DW-MRI was theoretically able to diagnose stroke in seconds [2]. Success

\footnotetext{
* Correspondence: zarrintanarmin@gmail.com

${ }^{4}$ Department of Radiology, Imam Reza Hospital, Tabriz University of Medical Sciences, Tabriz, Iran

Full list of author information is available at the end of the article
}

in these initiatives led researchers to focus on other applications of DW MRI in imaging, including oncoimaging [3]. Studies have shown that malignant tissue differs significantly from normal tissue in cellular architecture, cellular density, and extracellular matrix composition [4]. This causes the water molecules to have an altered diffusion pattern compared to normal tissue, which can be detected by DW-MRI. More so, different cancers arising from different tissues and cancers varying in stage also have different cellular structure, making them distinguishable in DW imaging [5]. Many studies have indeed shown this in cancers of the breast, brain, head and neck, colon, and the hepatobiliary system [6]. 
Many malignant and benign lesions comprising of cystic lesions, solid primary tumors, metastasis, patches of fibrosis, vascular lesions, and infectious lesions can be found in the liver and distinguishing them may be a diagnostic challenge [7, 8]. DW-MRI has shown beneficence in distinguishing the aforementioned lesions, but question remains regarding the sensitivity and specificity of this modality in diagnosing malignant lesions [9] and if DW MRI is able to distinguish between benign lesions of the liver and normal tissue. Previous studies have aimed to examine the efficacy of DW MRI in diagnosing liver masses, but no consensus exists regarding the routine use of DWI in detecting neoplastic lesions, as no definite cutoff point is set for values generated by DWI imaging, such as apparent diffusion coefficient (ADC) which is used for quantitative analysis of hepatic lesion [10]. Also, there are ambiguities regarding the optimal technical specifications for hepatic lesions, as different studies have reported DW images with varying $b$ values [11]. This study aims to study the sensitivity and specificity of DW imaging in diagnosing benign and malignant hepatic lesions and to examine whether an ADC value could be determined as a cutoff value to diagnose benign lesions from malignant ones.

\section{Methods}

\section{Patients}

In this prospective study, which was conducted between June 2016 and April 2019, in a tertiary referral center, a total of 93 patients with 118 liver masses were included. All patients had gone under initial evaluation by ultrasonography or dynamic CT scan of the liver. Then DWI MRI was performed for all patients. Inclusion criteria consisted of being above 18 years, having at least one lesion in the liver, having a definite pathologic diagnosis of the lesion (either by biopsy or fulfilling radiologic criteria), and being followed up for at least 6 months. Exclusion criteria consisted of patients who underwent chemotherapy before MRI imaging, inability of the patient to withstand the conditions necessary for performing an MRI, and lack of will to participate in the study.

In considering the results of ultrasonography, liver dynamic CT scan and biopsies, patients were categorized to five groups including liver hemangioma, liver focal nodular hyperplasia $(\mathrm{FNH})$, liver metastasis, cholangiocarcinoma, and hepatocellular carcinoma (HCC). Patients were also divided into two main groups of those with benign hepatic lesions (including liver hemangioma and FNH) and patients with malignant hepatic lesion (including liver metastasis, cholangiocarcinoma, and HCC). Other liver lesions were not included, such as hepatic fibrosis and infectious cysts or secondary hemorrhages in primary lesions, as our goal was to study the role of DW imaging in differentiating mass-like lesions which are not suitably diagnosed using ultrasonography and CT scan.

\section{Imaging technique}

MR imaging was performed utilizing 1.5-T MR systems (Siemens Medical Solutions, Erlangen, Germany) at the MRI ward of our medical educational center. Single-shot spin-echo (SE) echo-planar technique combined with fat suppression was performed according to these technical specifications: matrix $128 \times 128$, field of view $36 \mathrm{~cm} \times$ $36 \mathrm{~cm}$, section thickness $8 \mathrm{~mm}$, and gap $2 \mathrm{~mm}$ and scan time 3-4 min. The $b$ values of 0 and $1000 \mathrm{~s} / \mathrm{mm}^{-2}$ were applied in three orthogonal directions and combined to provide isotropic images. The ADC of the region of interest was calculated using workstation.

\section{Ethical considerations}

All patients being included in this study had signed written informed consent forms. This article was approved in the local ethics committee of the tertiary medical center in which it was performed. This study was performed according to the Helsinki declaration. Personal information, including imaging findings were not disclosed to any third party.

\section{Statistical analysis}

Statistical analysis was performed using Statistical Package for the Social Sciences (SPSS Version 19, Chicago, USA). Chi-square test was used to compare ADC values of malignant and benign lesions, while Student $T$ test was used to compare quantitative data. Power of the study was set at $80 \%$ and a $p$ value less than 0.05 was considered significant.

\section{Results}

Ninety three patients were included in this study. These patients had 118 distinct liver masses. The follow-up of the patients showed that 42 masses were malignant, while 76 where benign. Of all the patients, 73 had only a single lesion in their liver, of which 42 were malignant and 31 were benign, 15 and 5 patients had two and three lesions in their livers, respectively, which were all benign. Demographics of the patients and types of lesions are summarized in Table 1.

The highest ADC value was recorded for a hemangioma $\left(2.86 \times 10^{-3} \mathrm{~mm}^{2} / \mathrm{s}\right)$, while the lowest value recorded was for a metastasis $\left(0.49 \times 10^{-3} \mathrm{~mm}^{2} / \mathrm{s}\right)$. ADC values for five categories of hepatic lesions are summarized in Table 2. The mean ADC value of all lesions combined was $1.3364 \times 10^{-3} \mathrm{~mm}^{2} / \mathrm{s}$.

Table 1 Demographic information of patients being included in the study

\begin{tabular}{lllll}
\hline Tumor type & Number of lesions & Male & Female & Age \\
\hline Malignant lesion & 42 & 30 & 12 & $57.34 \pm 15.69$ \\
Benign lesion & 31 & 18 & 33 & $46.14 \pm 34 / 21$ \\
\hline
\end{tabular}


Table 2 Apparent diffusion coefficient values for detected lesions

\begin{tabular}{|c|c|c|c|c|c|c|c|c|}
\hline \multirow[b]{2}{*}{ Hemangioma } & \multirow{2}{*}{$\begin{array}{l}\text { Number } \\
\text { of lesions } \\
72\end{array}$} & \multirow{2}{*}{$\begin{array}{l}\text { Mean } \\
\text { ADC value* } \\
1.6134\end{array}$} & \multirow{2}{*}{$\begin{array}{l}\text { Standard } \\
\text { deviation } \\
0.34188\end{array}$} & \multirow{2}{*}{$\begin{array}{l}\text { Standard error } \\
0.04029\end{array}$} & \multicolumn{2}{|c|}{ Confidence interval (95\%) } & \multirow{2}{*}{$\begin{array}{l}\text { Minimum } \\
\text { ADC value * } \\
0.85\end{array}$} & \multirow{2}{*}{$\begin{array}{l}\text { Maximum } \\
\text { ADC value } \\
2.86\end{array}$} \\
\hline & & & & & 1.5331 & 1.6938 & & \\
\hline $\mathrm{FNH}$ & 4 & 1.1738 & 0.15593 & 0.07796 & 0.9256 & 1.4219 & 0.96 & 1.29 \\
\hline Metastasis & 31 & 0.8451 & 0.16475 & 0.02959 & 0.7847 & 0.9056 & 0.49 & 1.31 \\
\hline Cholangiocarcinoma & 7 & 0.9809 & 0.10885 & 0.04114 & 0.8802 & 1.0815 & 0.78 & 1.11 \\
\hline $\mathrm{HCC}$ & 4 & 0.9435 & 0.07911 & 0.03955 & 0.8176 & 1.0694 & 0.86 & 1.04 \\
\hline Total & 118 & 1.3364 & 0.45189 & 0.04160 & 1.2541 & 1.4188 & 0.49 & 2.86 \\
\hline
\end{tabular}

${ }^{*} A D C$ apparent diffusion coefficient $\left(10^{-3} \mathrm{~mm}^{2} / \mathrm{s}\right)$

The mean ADC values for benign and malignant lesions was $1.58 \pm 0.35\left(10^{-3} \mathrm{~mm}^{2} / \mathrm{s}\right)$ and $0.87 \pm 0.16\left(10^{-3}\right.$ $\mathrm{mm}^{2} / \mathrm{s}$ ), respectively. The mean ADC value was significantly higher in benign lesions $(p=0.001)$. ADC measurement had a sensitivity of $97.6 \%$ and specificity of $98.7 \%$ in differentiating malignant lesions from benign ones. Positive predictive value was $97.61 \%$ and negative predictive value was $98.68 \%$. Positive likelihood ratio was 75.07 , and negative likelihood ratio was 0.02 .

The cutoff value in differentiating benign and malignant lesions was $1.1 \times 10^{-3}$. Figure 1 shows the ROC curve for ADC values. Figure 2 shows mean ADC values for each of the five types of liver masses studied. Figure 3 shows the differences between the mean ADC values between the benign and malignant groups. Figures 4, 5, and 6 demonstrate examples of imaging findings.

\section{Discussion}

In the present study, the application of DW-MRI in differentiating malignant masses of the liver was examined. We found that DW-MRI was indeed a suitable method to distinguish malignant lesions and that ADC values

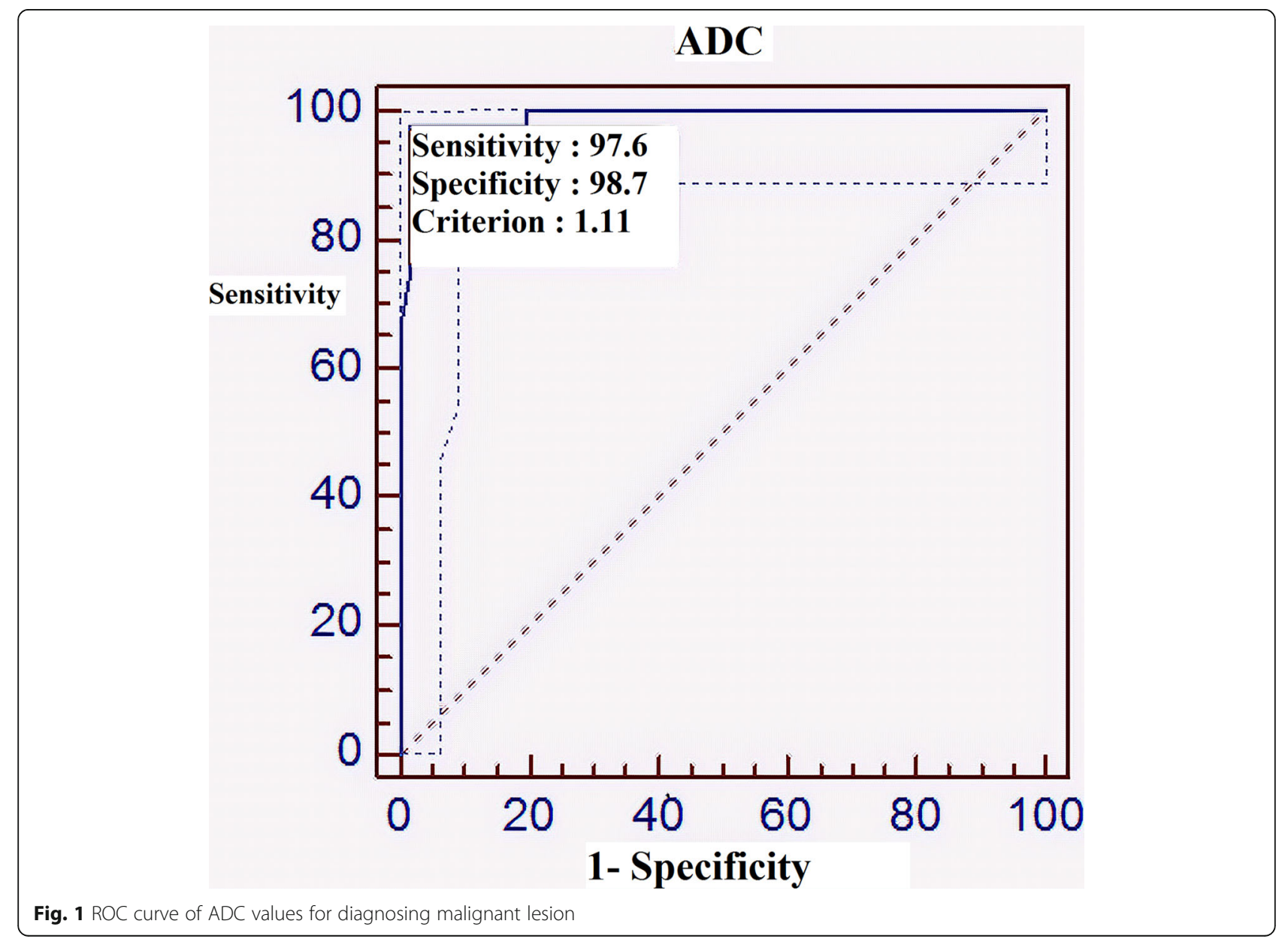




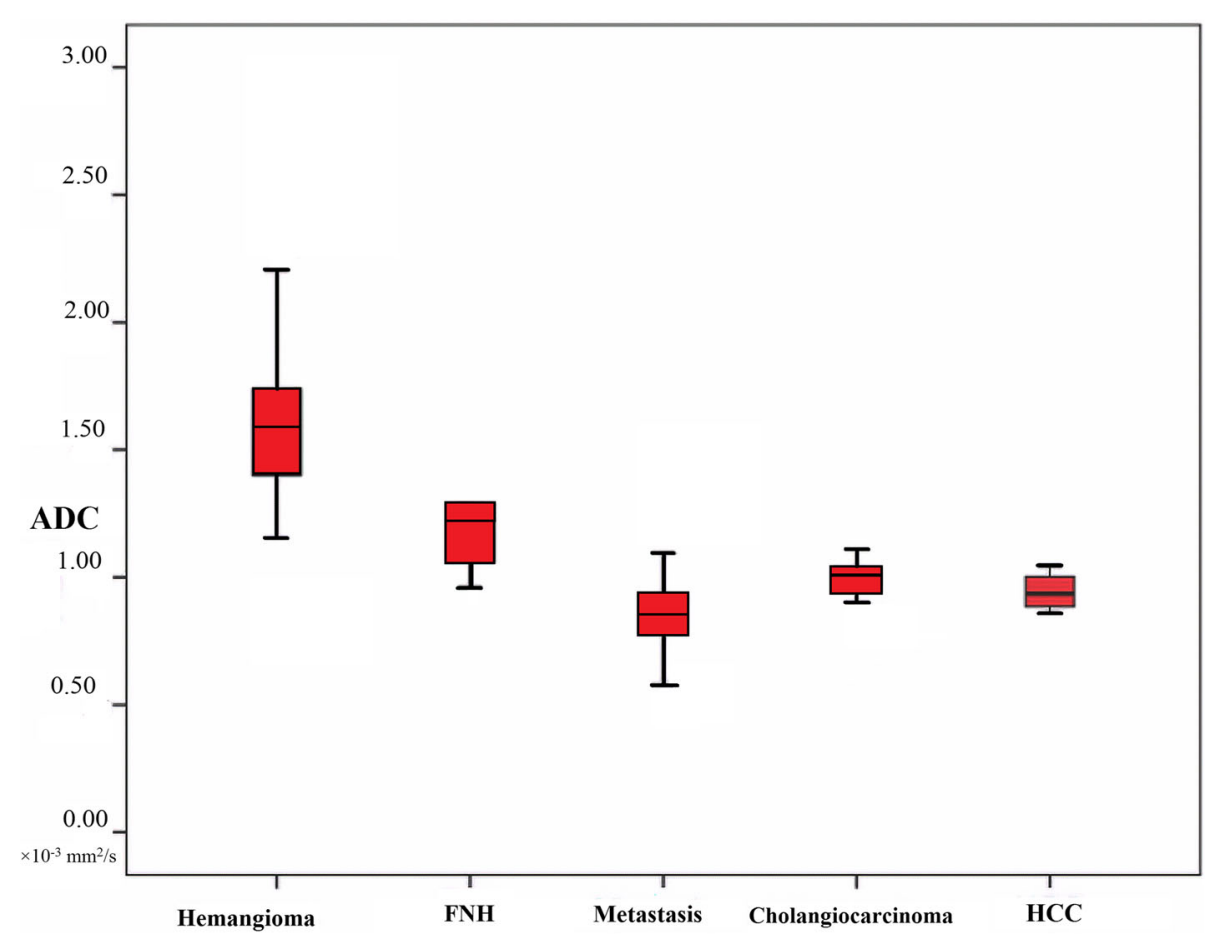

Fig. 2 Box and whisker plots of ADC values for different groups of liver masses

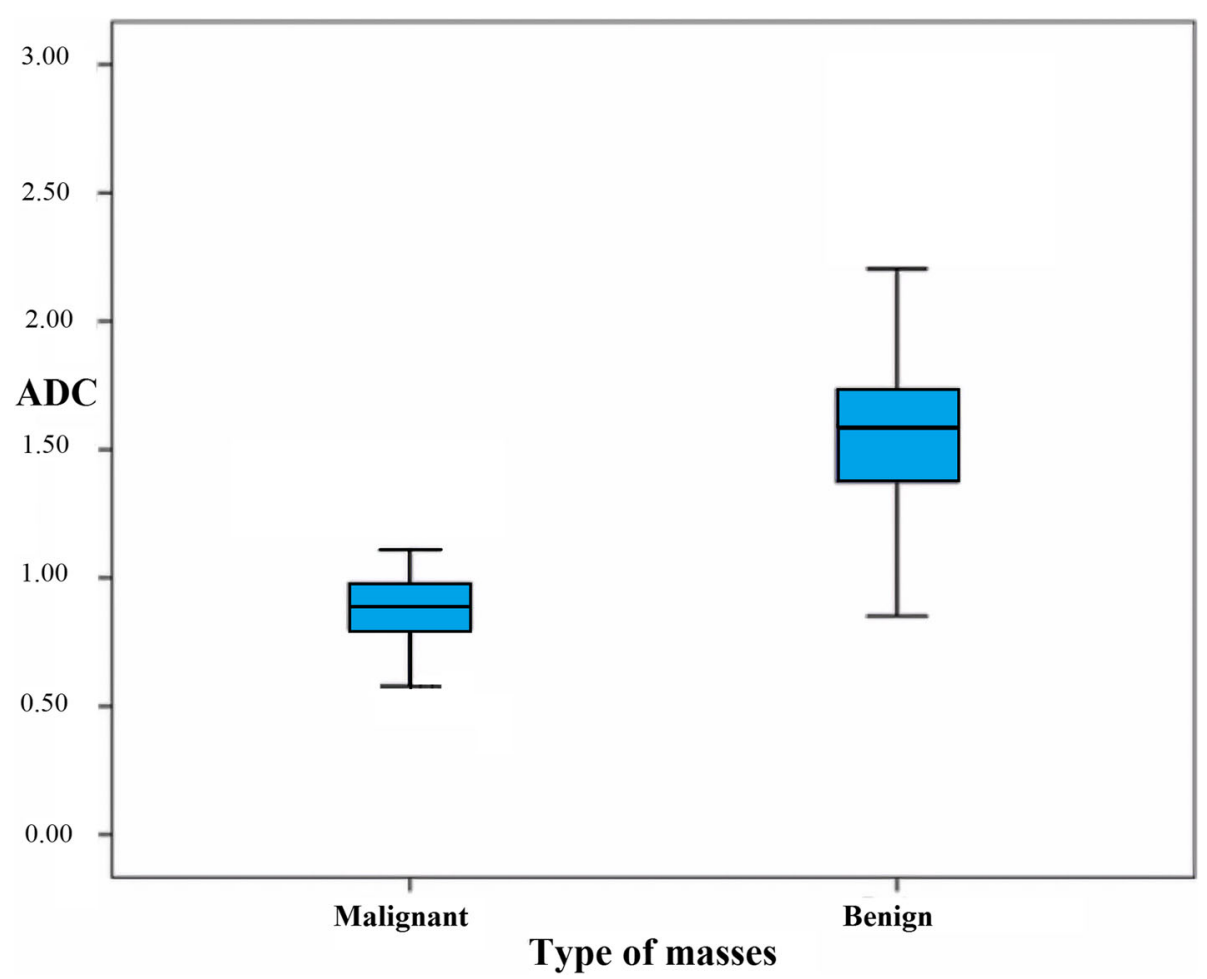

Fig. 3 Box and whisker plots of ADC values for malignant and benign lesions 


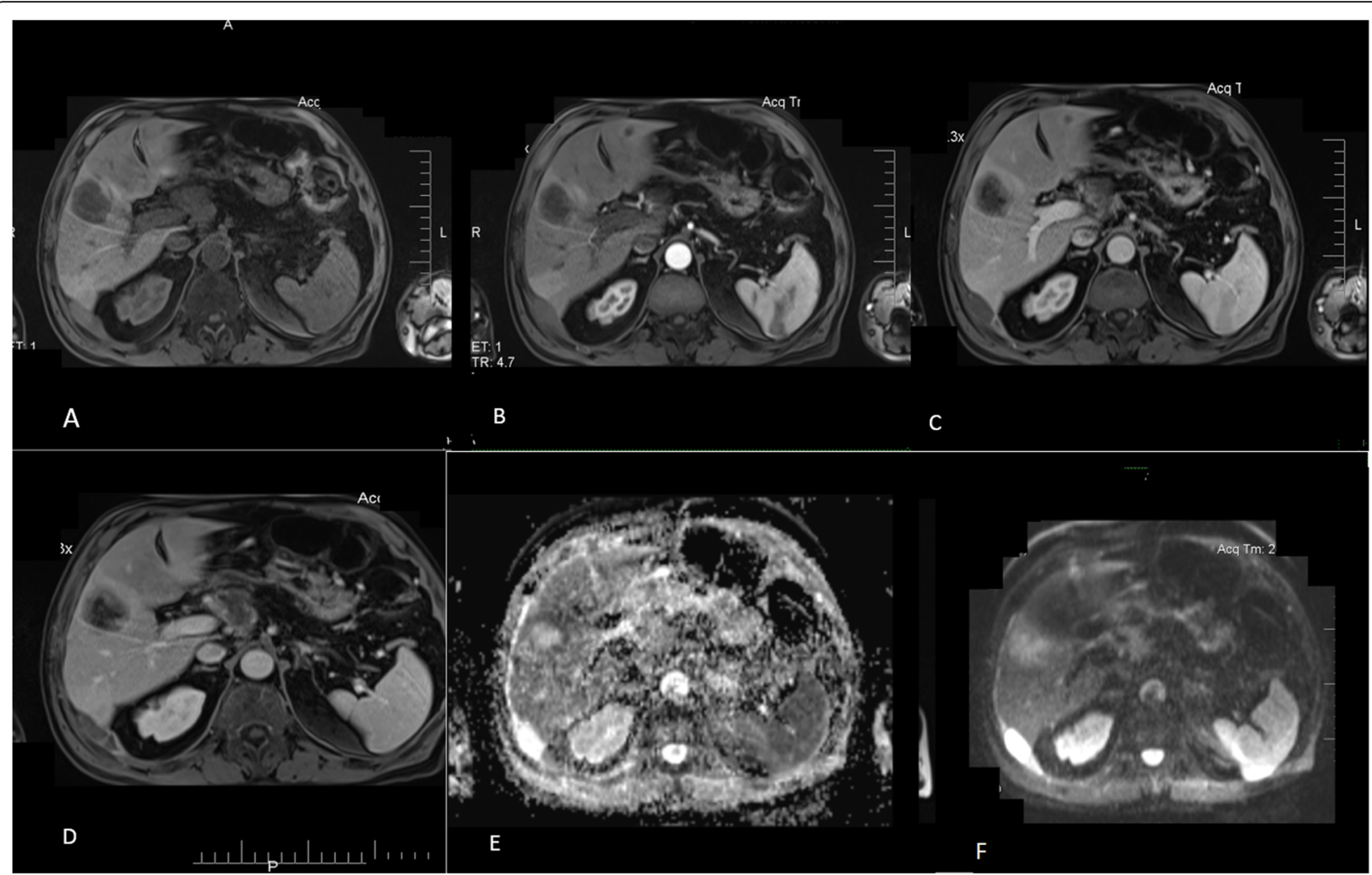

Fig. 4 Tri-phasic MRI of a patient with pancreatic cancer, presenting with liver metastasis. a pre-contrast T1 sequence. b Arterial phase T1 sequence. c venous phase T1 sequence. $\mathbf{d}$ Interstitial phase T1 sequence. As it can be seen, the lesion is hypo-intense in T1 sequences with evident ring enhancement. e ADC map showing restricted diffusion (ADC value:0.8 $\times 10^{-3} \mathrm{~mm}^{2} / \mathrm{s}$ ). $\mathbf{f}$ DWI imaging of the liver in $b$ value of $800 \mathrm{~s} / \mathrm{mm}^{2}$

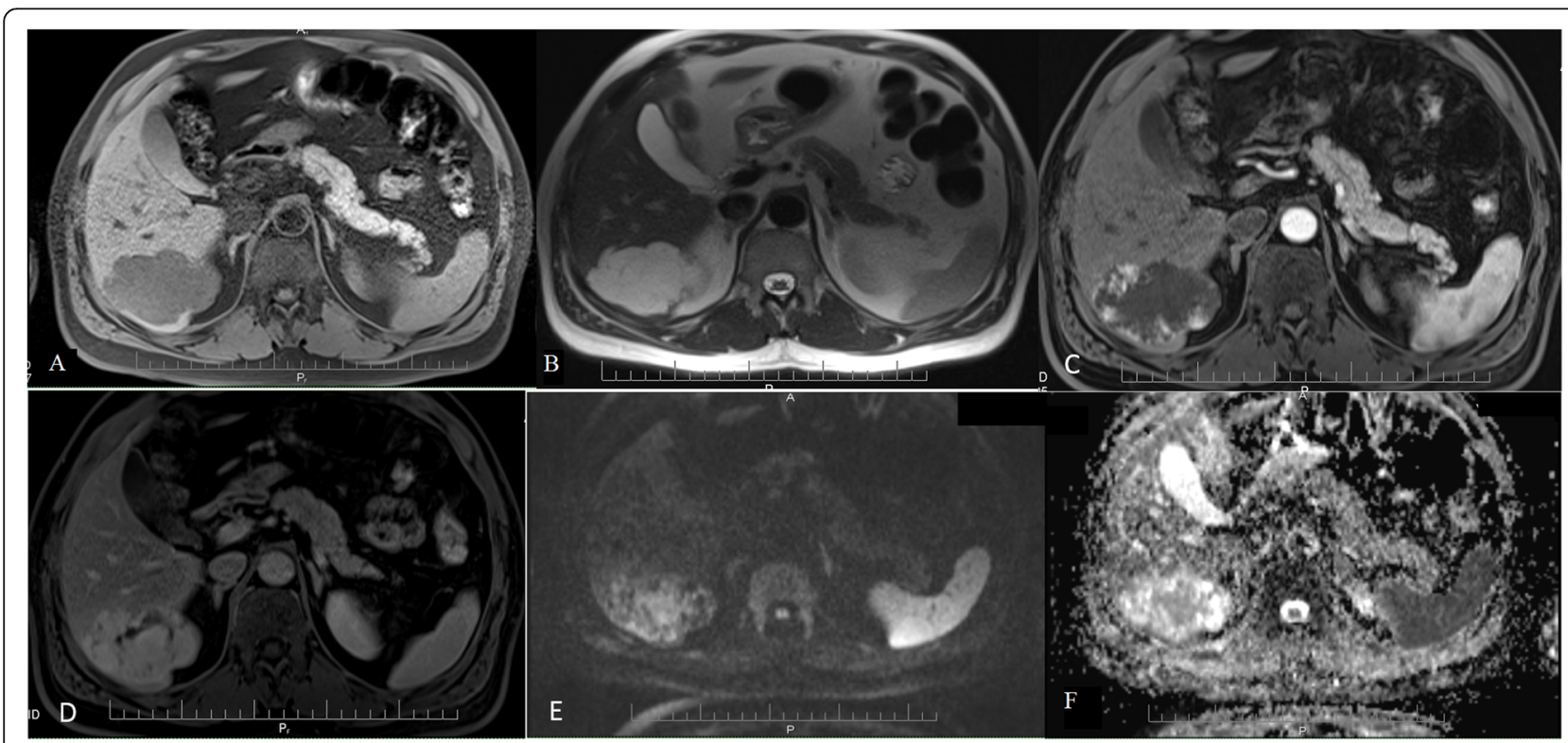

Fig. 5 a T1 sequence showing a hypo-intense lesion. $\mathbf{b}$ T2 HASTE sequence: There is a large oval mass at the right lobe of the liver, which is hyper intense compared to liver parenchyma. c Post gadolinium T1-weighted sequences at arterial phase. The image shows that the mass has nodular peripheral enhancement. d Post gadolinium T1-weighted sequences at delayed phase: The mass shows more peripheral centripetal enhancement compared to arterial phase. e DWI in $b$ value of $800 \mathrm{~s} / \mathrm{mm}^{2}$. The ADC value of the lesion was $1.53 \times 10^{-3} \mathrm{~mm}^{2} / \mathrm{s}$. $\mathbf{f ~ A D C ~ m a p ~ o f ~ t h e ~}$ liver. The aforementioned findings are suggestive of hemangioma 


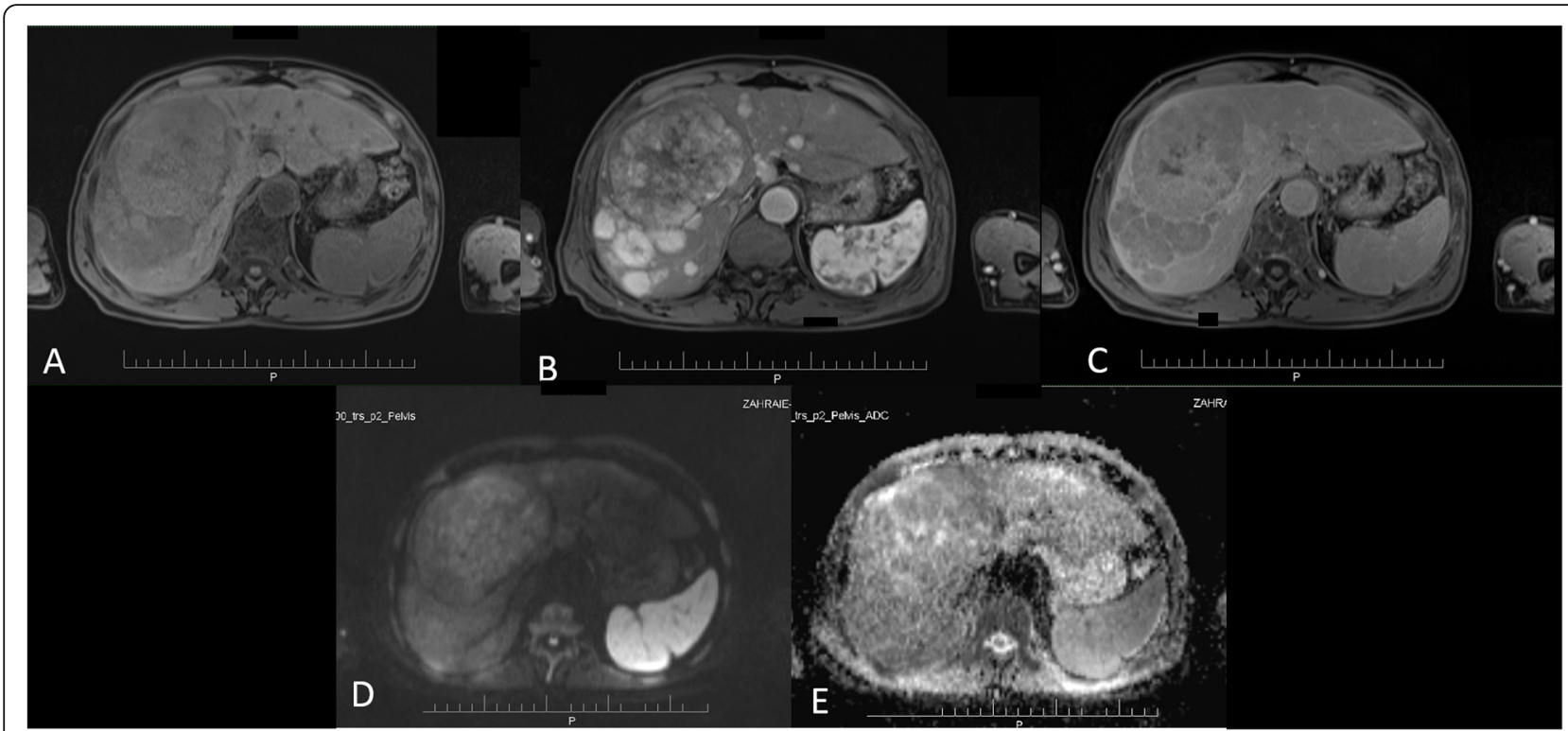

Fig. 6 An HCC in a 67-year-old patient with long standing cirrhosis. a The lesion in T1 with no contrast, shows a hypo intense mass. b Arterial phase, shows rapid hyper-enhancement. c Delayed phase, showing rapid washout. $\mathbf{d}$ DWI in $b$ value of $800 \mathrm{~s} / \mathrm{mm}^{2}$. The ADC value of the lesion equaled to $0.93 \times 10^{-3} \mathrm{~mm}^{2} / \mathrm{s}$. e ADC map of the liver

could even be used to distinguish specific lesions. Our results revealed that the ADC value is significantly higher in benign hepatic lesion compared to malignant ones. In this study, the cutoff point of $1.1 \times 10^{-3}$ was determined to differentiate between benign and malignant hepatic lesions.

Histopathologic studies have shown that various liver lesions have characteristic findings in pathological examination and unique cellular architecture. Malignant lesions such as hepatocellular carcinoma and metastasis are composed of a dense formation of cells tightly packed within an absent or minimal stroma, while focal nodular hyperplasia is composed of normal-looking hepatocytes imbedded in a rich fibrous mesh with considerably more extracellular space, and hemangiomas are composed of vascular spaces with an abundant stroma. These histopathologic variances could hypothetically be witnessed in DW MRI, as water molecules could move freely in less dense cellular structures such as those of benign lesions, resulting in increased values of ADC, which could be useful in differentiating between them and malignant lesions. This hypothesis could also be true for differentiating various malignant lesions from each other. This is shown best by grading of astrocytoma using ADC values [12].

Application of DW MRI in hepatic lesion detection is also possible. Initial results have been contradictory, and no definite suggestions have been made, but primary results have shown that low $b$ values could be beneficial in lesion detection, while increasing the $b$ value could assist in lesion classification.
Experimental evidence in this regard is given by Testa et al., where DW MRI was used to classify 188 masses in 67 adults. Of all the lesions, 105 were metastatic lesions, 37 were hemangiomas, 42 were cysts, 2 were adenomas, and 2 were nodular hyperplasia. This study showed that the average ADC for metastasis was $1.0 \times 10^{-3} \mathrm{~mm}^{2} / \mathrm{s}$ (CI $0.8-1.3$ ), $1.4 \times 10^{-3} \mathrm{~mm}^{2} / \mathrm{s}$ (CI 1.1-1.7) for solid benign lesions and $2.4 \times 10^{-3} \mathrm{~mm}^{2} / \mathrm{s}(\mathrm{CI} 2.1-2.6)$ for cystic lesions. The cutoff value for distinguishing metastatic lesions from benign solid lesions was reported to be $1.2 \times$ $10^{-3} \mathrm{~mm}^{2} / \mathrm{s}$, and $1.5 \times 10^{-3} \mathrm{~mm}^{2} / \mathrm{s}$ for all benign lesions, including cysts. The accuracy of DW MRI in detecting malignant lesions from non-malignant solid lesions was $71 \%$, and $78 \%$ if cysts were included. A study performed by Kilickesmez et al. also studied the efficacy of DW MRI in diagnosing malignant lesions from benign lesions. They included benign entities such as simple liver cysts, hydatid cysts, and hemangiomas. Most of the lesions included were of the aforementioned, while a smaller number of malignant lesions were also included. The authors suggested that there was a significant difference between the ADC values of hepatic lesions and normal liver parenchyma, and also between hemangiomas and HCCs or metastasis [13]. This study provides practical evidence regarding the use of DW in liver masses, but the low number of malignant lesions, and high number of normal liver tissue limits its generalizability (65 normal liver specimens were included compared to 13 metastases and $13 \mathrm{HCCs}$ ). This study does not include FNHs and cholangiocarcinomas, entities which theoretically would have closer ADC 
values to the mean $\mathrm{ADC}$ value of malignant lesions, compared to the ADC value of cystic lesions (which is considerably higher).

In regard to previous studies, it is noteworthy to mention that diagnosing cystic lesions is not of a challenge as those of diagnosing solid benign and malignant masses, and not including cysts could reveal the true efficacy of DW imaging in detecting liver lesions. The same could also be said regarding fibrotic lesions of the liver, which have distinct radiological appearances [14].

A large number of malignant hepatic lesions are metastasis from distant sites and differentiating between these lesions and non-malignant hepatic lesions is of great importance, especially in patients effected by a primary tumor in another organ. Parikh et al. found that there was no significant difference between the ADC values of benign hepatic lesions, metastatic lesions, and $\mathrm{HCC}\left(\mathrm{ADC}=1.49 \pm 0.49 \times 10^{-3} \mathrm{~mm}^{2} / \mathrm{s}, 1.50 \pm 0.42 \times\right.$ $10^{-3} \mathrm{~mm}^{2} / \mathrm{s}$, and $1.31 \pm 0.33 \times 10^{-3} \mathrm{~mm}^{2} / \mathrm{s}$ ) [15]. In our study, there was a significant difference between the ADC values of non-malignant lesions and metastasis. This could be because of utilizing $b$ values up to 1000 , compared to Parikh et al. where only $b$ values of 0,100 , and 500 were used. Also, use of non-DWI MRI imaging could be beneficial in increasing the sensitivity of MRI in detecting malignant metastatic lesions.

A meta-analysis by Xia et al. published in 2010 focused on the efficacy of DW MRI in diagnosing hepatic lesions, and reported that indeed DW MRI would be of beneficence, but the exact sensitivity and specificity would vary greatly based on the diagnostic implication, as differentiating between normal hepatic masses and metastasis would be easier than differentiating focal nodular hyperplasia from low-grade hepatocellular carcinoma. The authors suggested that more studies would be needed to determine exact imaging protocols incorporating DW MRI, and that more studies were needed to determine the efficacy in differentiating various malignant lesions from each other [16]. In our study, metastasis, HCC, and cholangiocarcinoma had almost identical ADC values, which was in concordance to other similar studies [17].

Ogihara et al. [18] utilized DW MRI to grade HCCs and examined which variables could be used to differentiate between high-grade and low-grade HCCs. They studied 83 patients with 100 histologically diagnosed HCCs. They used DW MRI with a $b$ value of 1000 and retrospectively matched radiologic findings with histopathologic findings. They found that in $b$ values of 800 and 1000, ADC was not significantly different between well and poorly differentiated HCCs and it could only be used in differentiating well differentiated HCCs from moderately and poorly differentiated HCCs, while relative contrast ratio (RCR) and contrast-to-noise ratio (CNR) could be used to differentiate between all three differentiations of HCC. Previous studies also had hinted that non-ADC values could be utilized in determining the differentiation of tumors, but no consensus exists regarding the best strategy to determine the grading of malignant tumors based on ADC or any other parameters $[19,20]$.

As mentioned, the growing role of DWI MRI has been encountered with challenges, including technical difficulties and limitations. T2 shine effect is defined as the hyper intensity of tissue with normal diffusion in DW imaging which is caused by long T2 decay time. T2 shine effect depends on the intrinsic characteristics of the lesions being imaged, and can depend on cellular structure, lesion size, and even anatomical location of the lesion [21]. This effect is especially important in lesions such as hemangiomas, where DW imaging may mislead towards hyper-cellular lesions, including malignant lesions. Experimental studies have shown that increasing the $b$ value may increase the contribution of cellularity of the lesion to signal intensity, while decreasing that of T2 shine effect [22].

More challenges may also arise in utilizing DW imaging alongside previously established imaging guidelines, such as Liver Imaging Reporting and Data System (LI-RADS) [23]. This imaging guideline is effectively introduced in order to enable radiologists to distinguish HCC from non-malignant lesions in patients with chronic conditions such as cirrhosis which are regarded as risk factors for HCC, and no further interpretation is suggested for distinguishing other malignant lesions (excluding lesions such as confluent hepatic fibrosis which may be classified as possibly malignant lesions) [24]. In contrast, DW is used to distinguish all sorts of malignant lesions from benign ones, and as mentioned, no consensus exists regarding its role in the diagnosis of $\mathrm{HCC}$ alone, as a clinical entity. In this study, we not only included patients with established risk factors for HCC, but also included those with otherwise no clinically proven risk factor, who had a liver mass with an unknown diagnosis. Serious questions remain regarding the efficient use of DW alongside these guidelines (cost efficacy, specificity of combined diagnostic plans and availability issues), but evidence suggests that DW could be used as a supplementary diagnostic technique in LR3-5 lesions, and can also increase the sensitivity of the LI-RADS in detecting HCC lesions [25, 26].

The present study was conducted in a single tertiary center, which limits the extent to which the results could be generalized. In this study, we concluded only five subgroups of hepatic lesions, which had similar appearances in CT scan and sonographic imaging, so our results do not indicate the beneficence of DW imaging in diagnosing other sorts of liver pathologies, including cysts and fibrotic lesions. If these lesions were included, a dramatic 
change may have occurred in mean ADC values of benign lesions, and the analysis may have yielded a different result. More so, some lesions with borderline ADC values such as secondary hemorrhages in adenomas and high-density cysts were not included, which may further limit generalizability.

\section{Conclusion}

DW MRI can differentiate benign and malignant hepatic lesions based on ADC values calculated in various $b$ values, especially high $b$ values. More so, different hepatic lesions have a distinct mean ADC profile which can be used to further differentiate subgroups of benign and malignant lesions. Importantly, non-ADC parameters of DW imaging could also be used in this regard, although our study does not focus on this topic. More studies performed on hepatic lesions could better determine the mean ADC values for various lesions, and future metaanalysis of these studies could determine clear, evidencebased cutoffs for differentiating various lesions.

\section{Abbreviations}

ADC: Apparent diffusion coefficient; DWl: diffusion-weighted imaging; FNH: Focal nodular hyperplasia; HCC: Hepatocellular carcinoma; MRI: Magnetic resonance imaging

\section{Acknowledgements}

We would like to thank the radiology staff of the medical educational center in which the case underwent diagnostic imaging. This study was done as a thesis for receiving a specialty degree in radiology, done by Dr. Roghayeh Razzaghi. This study was in part supported by Tabriz University of Medical Sciences.

\section{Authors' contributions}

$R J, A S B$, and MKT did the data collection, manuscript preparation, approval of the final version, interpretation of imaging findings and study conception. RR and $\mathrm{J}$ did the data collection, obtaining the informed consent, study conception, and approval of the final version of the study. SH did the data collection, patient selection, and approval of the final version of the study. MMAA did the study conception, manuscript preparation, systemic search of the literature, statistical analysis, and approval of the final version of the study. AKN did the data collection, manuscript preparation, statistical analysis, and approval of the final version of the study. AZ did the data collection, manuscript preparation, study conception, and approval of the final version of the manuscript.

\section{Funding}

This study did not receive any funding, other than that provided by Tabriz University of Medical Sciences.

\section{Availability of data and materials}

All data and materials are available based on reasonable request, according to the guidelines of the institution in which the study was approved, and based on the guidelines regarding sharing patient information.

\section{Ethics approval and consent to participate}

The study was approved by the ethical board of the medical educational center in which it was performed (No. 1396-10-1/14). The patients being included had signed written informed consent notes. Furthermore, all patients are informed that they may be subjects of scientific experiments, and their rights in this regard.

\section{Consent for publication}

Oral and written consents were taken from the patient and one of the legal representatives (in this case, a family member).

\section{Competing interests}

The authors declare that they have no competing interests.

\section{Author details}

'Radiology Department, Medical Radiation Sciences Research Group, Imam Reza Hospital, Tabriz University of Medical Sciences, Tabriz, Iran. ${ }^{2}$ General and Vascular Surgery Department, Imam Reza Hospital, Tabriz University of Medical Sciences, Tabriz, Iran. ${ }^{3}$ Aging Research Institute, Tabriz University of Medical Sciences, Tabriz, Iran. ${ }^{4}$ Department of Radiology, Imam Reza Hospital, Tabriz University of Medical Sciences, Tabriz, Iran.

Received: 21 October 2019 Accepted: 23 January 2020

Published online: 02 March 2020

\section{References}

1. Song KD, Kim SH, Lim HK, Jung S-H, Sohn I, Kim HS (2015) Subcentimeter hypervascular nodule with typical imaging findings of hepatocellular carcinoma in patients with history of hepatocellular carcinoma: natural course on serial gadoxetic acid-enhanced MRI and diffusion-weighted imaging. Eur Radiol 25(9):2789-2796

2. Okorie CK, Ogbole Gl, Owolabi MO, Ogun O, Adeyinka A, Ogunniyi A (2015) Role of diffusion-weighted imaging in acute stroke management using lowfield magnetic resonance imaging in resource-limited settings. West Afr J Radiol. 22(2):61-66

3. Padhani AR, Koh D-M, Collins DJ (2011) Whole-body diffusion-weighted MR imaging in cancer: current status and research directions. Radiology. 261(3):700-718

4. He L, Long LR, Antani S, Thoma GR (2012) Histology image analysis for carcinoma detection and grading. Comp Methods Programs Biomed. 107(3):538-556

5. Chilla GS, Tan CH, Xu C, Poh CL (2015) Diffusion weighted magnetic resonance imaging and its recent trend-a survey. Quantitative imaging in medicine and surgery. 5(3):407

6. Malayeri AA, El Khouli RH, Zaheer A, Jacobs MA, Corona-Villalobos CP, Kamel IR et al (2011) Principles and applications of diffusion-weighted imaging in cancer detection, staging, and treatment follow-up. Radiographics. 31(6):1773-1791

7. Robinson P (2002) Indeterminate liver lesions in cancer. Cancer Imaging. 2(2):130

8. Mergo PJ, Ros PR (1998) Benign lesions of the liver. Radiologic Clinics of North America. 36(2):319-331

9. Saito K, Tajima Y, Harada TL (2016) Diffusion-weighted imaging of the liver: current applications. World journal of radiology. 8(11):857

10. Hasan NMA, Zaki KF, Alam-Eldeen MH, Hamedi HR (2016) Benign versus malignant focal liver lesions: diagnostic value of qualitative and quantitative diffusion weighted MR imaging. Egypt J Radiol Nucl Med. 47(4):1211-1220

11. Yang D-w, Wang K-y, Yao X, Ye H-Y, Jiang T, Liu Y et al (2016) Diffusionweighted imaging with two different b-values in detection of solid focal liver lesions. BioMed Res Int 2016:8128207

12. Darbar A, Waqas M, Enam SF, Mahmood SD (2018) Use of preoperative apparent diffusion coefficients to predict brain tumor grade. Cureus 10(3):e2284-e

13. Kilickesmez O, Bayramoglu S, Inci E, Cimilli T (2009) Value of apparent diffusion coefficient measurement for discrimination of focal benign and malignant hepatic masses. Journal of medical imaging and radiation oncology. 53(1):50-55

14. Obmann VC, Mertineit N, Berzigotti A, Marx C, Ebner L, Kreis R et al (2018) CT predicts liver fibrosis: prospective evaluation of morphology- and attenuation-based quantitative scores in routine portal venous abdominal scans. PloS one. 13(7):e0199611

15. Parikh T, Drew SJ, Lee VS, Wong S, Hecht EM, Babb JS et al (2008) Focal liver lesion detection and characterization with diffusion-weighted MR imaging: comparison with standard breath-hold T2-weighted imaging. Radiology. 246(3):812-822

16. Xia D, Jing J, Shen H, Wu J (2010) Value of diffusion-weighted magnetic resonance images for discrimination of focal benign and malignant hepatic lesions: a meta-analysis. J Magn Resonan Imaging. 32(1):130-137

17. Ergelen R, Sahin C, Bal H, Tuney D (2016) Diffusion-weighted MRl: in differential diagnosis of liver masses

18. Ogihara Y, Kitazume Y, Iwasa Y, Taura S, Himeno Y, Kimura T et al (2018) Prediction of histological grade of hepatocellular carcinoma using 
quantitative diffusion-weighted MRI: a retrospective multivendor study. The British journal of radiology. 91(1084):20170728

19. Nakanishi M, Chuma M, Hige S, Omatsu T, Yokoo H, Nakanishi K et al (2012) Relationship between diffusion-weighted magnetic resonance imaging and histological tumor grading of hepatocellular carcinoma. Annals of surgical oncology. 19(4):1302-1309

20. Le Moigne F, Boussel L, Haquin A, Bancel B, Ducerf C, Berthezene $Y$ et al (2014) Grading of small hepatocellular carcinomas $(\leq 2 \mathrm{~cm})$ : correlation between histology, $\mathrm{T} 2$ and diffusion-weighted imaging. $\mathrm{Br} J$ Radiol. 87(1041):20130763

21. Duran R, Ronot M, Kerbaol A, Van Beers B, Vilgrain V (2014) Hepatic hemangiomas: factors associated with $\mathrm{T} 2$ shine-through effect on diffusionweighted MR sequences. Eur J Radiol 83(3):468-478

22. Inan N, Kilinc F, Sarisoy T, Gumustas S, Akansel G, Demirci A (2010) Diffusion weighted MR imaging in the differential diagnosis of haemangiomas and metastases of the liver. Radiol Oncol. 44(1):24-29

23. Chernyak V, Fowler KI, Kamaya A, Kielar AZ, Elsayes KM, Bashir MR et al (2018) Liver Imaging Reporting and Data System (LI-RADS) version 2018: imaging of hepatocellular carcinoma in at-risk patients. Radiology. 289(3):816-830

24. Faria SC, Ganesan K, Mwangi I, Shiehmorteza M, Viamonte B, Mazhar S et al (2009) MR imaging of liver fibrosis: current state of the art. Radiographics. 29(6):1615-1635

25. Patella F, Pesapane F, Fumarola EM, Emili I, Spairani R, Angileri SA et al (2018) CT-MRI LI-RADS v2017: a comprehensive guide for beginners. J Clin Transl Hepatol 6(2):222

26. Basha MAA, Refaat R, Mohammad FF, Khamis ME, El-Maghraby AM, El Sammak AA et al (2019) The utility of diffusion-weighted imaging in improving the sensitivity of LI-RADS classification of small hepatic observations suspected of malignancy. Abdom Radiol 44(5):1773-1784

\section{Publisher's Note}

Springer Nature remains neutral with regard to jurisdictional claims in published maps and institutional affiliations.

\section{Submit your manuscript to a SpringerOpen ${ }^{\circ}$ journal and benefit from:}

- Convenient online submission

- Rigorous peer review

- Open access: articles freely available online

- High visibility within the field

- Retaining the copyright to your article

Submit your next manuscript at $\boldsymbol{\nabla}$ springeropen.com 